\title{
Original Research Communications
}

\section{Short-term variability in body weight predicts long-term weight gain ${ }^{1}$}

\author{
Michael R Lowe, ${ }^{2 *}$ Emily H Feig, ${ }^{2}$ Samantha $R$ Winter, $^{2}$ and Eric Stice ${ }^{3}$ \\ ${ }^{2}$ Drexel University, Philadelphia, PA; and ${ }^{3}$ Oregon Research Institute, Eugene, OR
}

\begin{abstract}
Background: Body weight in lower animals and humans is highly stable despite a very large flux in energy intake and expenditure over time. Conversely, the existence of higher-than-average variability in weight may indicate a disruption in the mechanisms responsible for homeostatic weight regulation.

Objective: In a sample chosen for weight-gain proneness, we evaluated whether weight variability over a 6-mo period predicted subsequent weight change from 6 to 24 mo.

Design: A total of 171 nonobese women were recruited to participate in this longitudinal study in which weight was measured 4 times over 24 mo. The initial 3 weights were used to calculate weight variability with the use of a root mean square error approach to assess fluctuations in weight independent of trajectory. Linear regression analysis was used to examine whether weight variability in the initial 6 mo predicted weight change 18 mo later. Results: Greater weight variability significantly predicted amount of weight gained. This result was unchanged after control for baseline body mass index (BMI) and BMI change from baseline to 6 mo and for measures of disinhibition, restrained eating, and dieting.

Conclusions: Elevated weight variability in young women may signal the degradation of body weight regulatory systems. In an obesogenic environment this may eventuate in accelerated weight gain, particularly in those with a genetic susceptibility toward overweight. Future research is needed to evaluate the reliability of weight variability as a predictor of future weight gain and the sources of its predictive effect. The trial on which this study is based is registered at clinicaltrials.gov as NCT00456131. Am J Clin Nutr 2015;102:995-9.
\end{abstract}

Keywords: body weight, set point, weight variability, weight fluctuations, weight gain

\section{INTRODUCTION}

The high prevalence of obesity and the difficulty in treating it successfully indicate that more emphasis is needed on obesity prevention. The cost-effectiveness of obesity prevention interventions should be enhanced if they can be targeted at those who do not have a weight problem but are likely to gain weight in the future.
Body weight in humans and lower animals is usually regulated with great precision in an automatic fashion (1). For instance, over a 1-y period, the energy flux (number of calories consumed and expended) experienced by an average-size human being is tremendous (roughly 700,000 kcal), yet human body weight usually varies very little over long periods of time. This high degree of weight stability, which historically has been achieved in the absence of conscious effort, indicates the operation of a highly precise system of energy balance regulation (1).

By the same token, greater-than-average variability in body weight over time may indicate that the body weight regulatory system is being undermined by biological or environmental influences. Further, if heightened weight variability occurs in the midst of an obesogenic environment, it may be an early warning sign that a given individual's body weight regulatory system is being influenced by orexigenic influences in the environment. In addition to weight gain, weight variability could be increased by individuals' attempts to lose weight to limit or reverse weight gains, especially in young women who are particularly sensitive to changes in their weight (2). Thus, the existence of elevated weight fluctuations over time could signal that a previously effective body weight regulatory system is being degraded in its ability to automatically adjust energy intake and output to maintain a high degree of stability in body weight.

As part of a project testing a weight-gain prevention intervention, we collected longitudinal data on nearly 300 female college freshmen whose BMIs were primarily in the healthy weight range. The participants were selected to be both prone to and concerned about weight gain. Participants' body weights were measured at 4 time points over a 2-y period. The weight-gain prevention intervention had no detectable effect on weight change, but the data set provided an opportunity to test, in a primarily normal-weight sample that was vulnerable to weight gain, whether degree of variability in body weight over an initial time span of 6 mo pre-

\footnotetext{
${ }^{1}$ Based on research supported by NIH grant DK072982.

*Towhom correspondence should be addressed. E-mail: lowe@drexel. edu.

Received May 21, 2015. Accepted for publication August 5, 2015.

First published online September 9, 2015; doi: 10.3945/ajcn.115.115402.
} 
dicted susceptibility to weight gain over a subsequent 18-mo period. We hypothesized that higher weight variability would predict greater subsequent weight gain. This relation was hypothesized to hold when absolute changes in weight over the 6-mo period were controlled for. Finally, we tested whether, if the predicted effect was obtained, consummatory patterns reflecting disinhibitory eating or restrained eating could account for the results.

\section{METHODS}

\section{Subjects}

Participants were 294 female first-year students [age: $18.24 \pm$ $0.44 \mathrm{y}$; BMI (in $\mathrm{kg} / \mathrm{m}^{2}$ ): $23.65 \pm 2.88$; all values are expressed as means \pm SDs unless otherwise indicated] recruited from 2 universities in Philadelphia. They were volunteers in a randomized, controlled weight gain prevention trial. Participants with a history of eating disorder were excluded. All participants were required to have one or more of the following characteristics, which were expected to confer an elevated risk of weight gain: selfreported history of dieting (3), above-average body dissatisfaction (4), or elevated weight suppression (5). The sample was $17.7 \%$ Asian/Pacific Islander, 11.4\% African American, 5.5\% Hispanic, $57.8 \%$ European American, and 7.6\% other/mixed racial heritage.

\section{Subsample subjects}

Of all participants in the study, 171 had their body weights measured at baseline, 6-wk, 6-mo, and 2-y assessments. These composed the primary sample reported here. However, we also conducted multiple imputation for those with one or more missing values so the analyses could be reanalyzed with all participants (details provided below). This subsample of 171 did not differ significantly from the group that did not provide weights at all assessments $(n=123)$ in terms of age or any predictor variables in the analyses. At the initial study visit, participants in this subsample were of typical college age $(18.27 \pm 0.47 \mathrm{y}$; range: $17-20 \mathrm{y})$ and had a normal BMI on average (23.55 \pm 2.67 ; range: 19.36-35.19). A minority (19.3\%) reported currently dieting to lose weight. Over the first 6 mo of the study, weights increased slightly and nonsignificantly on average (mean: $0.27 \mathrm{~kg}$ ), but there was high variability in weight change (SD: $3.17 \mathrm{~kg}$, range: -19.17 to $9.40 \mathrm{~kg})$. Among those who gained weight $(n=96)$, mean weight gain was $2.27 \pm 1.99 \mathrm{~kg}$. Among those who lost weight $(n=75)$, mean weight loss was $2.29 \pm 2.49 \mathrm{~kg}$. A similar pattern was seen over the $2 \mathrm{y}$ of the study, with mean weights increasing by only $0.34 \mathrm{~kg}$ on average, but weight change ranging from -13.20 to $29.20 \mathrm{~kg}$, resulting in a high degree of weight variability $(\mathrm{SD}=5.06)$. Among the 89 participants who gained weight over $2 \mathrm{y}$, they gained on average $3.90 \pm 4.11 \mathrm{~kg}$. Among the 82 who lost weight, they lost on average $3.43 \pm 2.80 \mathrm{~kg}$.

\section{Methods}

Participants were recruited for a study the purpose of which was to test the effectiveness of a weight-gain prevention intervention over $2 \mathrm{y}$. After a baseline assessment, participants were randomly assigned to an experimental intervention or an assessment-only control condition. The intervention was conducted in 6 weekly, 1-h, closed group sessions, with 6-8 participants/group. The intervention comprised nutrition education and techniques for instigating behavior change (6) and components from Lowe's nutrition-focused weight-control program $(7,8)$. Lowe's Nutritrol program teaches methods for reducing portion sizes and the energy density of the diet and gaining greater control over the personal food environment. The intervention and control groups did not significantly differ in weight change (BMI change in control and experimental groups were -0.22 and +0.24 units, respectively) or on other outcomes over the course of the study, thus the sample was collapsed across group assignment for the subsequent analyses.

\section{Measures}

Assessments were conducted by an assessor who was blind to treatment condition.

\section{Measured height and weight}

Participants were weighed without shoes and in light clothing with the use of an electronic scale that measured to the nearest $0.01 \mathrm{~kg}$. Height was measured to the nearest $0.01 \mathrm{~cm}$ with a stadiometer. BMI was calculated for each participant. BMI correlates with dual-energy X-ray absorptiometry-measured body fat ( $r=0.80-0.90)$ and health measures such as adverse lipoprotein profiles (9). BMI has been found to be a sensitive measure of adiposity that is adjusted for variation in height.

\section{Disinhibition}

The Three-Factor Eating Questionnaire-Disinhibition subscale (TFEQ-D) ${ }^{4}$ (10) was administered to measure overeating in response to cognitive, emotional, and environmental cues. This scale has 16 items, and higher scores indicate greater disinhibition. The scale shows high internal consistency and testretest reliability (10) and predicted laboratory-based eating (11), stress-induced eating (12), and weight gain (13).

\section{Restrained eating and dieting}

\section{Dutch Restrained Eating Scale}

Restrained eating was measured with the Dutch Restrained Eating Scale (DRES) (14). Participants rated the frequency with which they try to restrict their food intake with the use of 5-point scales ranging from "never" to "always." This scale has high internal consistency (Cronbach's $\alpha=0.95$ ) and test-retest reliability $(r=0.82)(14)$, and it is related to reduced laboratory food intake (15). Although restraint scales have not been found to be related to measures of naturalistic caloric intake (16), it is thought that restrained eaters fluctuate between dieting and not dieting and that such measures could therefore be related to weight variability (17).

\section{Revised Restraint Scale}

Restrained eating was also measured with the 10-item Revised Restraint Scale (RRS) (18). This scale has high internal consistency (Cronbach's $\alpha=0.78-0.86$ ) and test-retest reliability $(r=0.95)(19)$, and it predicts counter-regulatory eating in laboratory eating tests $(18,20)$.

\footnotetext{
${ }^{4}$ Abbreviations used: DRES, Dutch Restrained Eating Scale; RMSE, root mean square error of variation; RRS, Revised Restraint Scale; TFEQ-D, Three-Factor Eating Questionnaire-Disinhibition subscale.
} 


\section{Dieting status}

Weight loss dieting has been found to reliably predict future weight gain (3). We assessed dieting by asking whether participants were "currently dieting to lose weight."

\section{Ethics}

This study was approved by the Institutional Review Board at Drexel University in Philadelphia. Voluntary informed written consent was obtained from all study participants.

\section{Statistics}

All analyses were conducted with the subsample of study completers $(n=171)$ and were then re-estimated by using a multiple imputation technique to generate values for the missing cases. The multiple imputation technique in SPSS uses a "Fully Conditional Specification" method based on an iterative Markov chain Monte Carlo procedure. A linear regression model was fitted based on measured weights, and it was then used to impute the missing observations at each of the 4 assessments. All analyses were then conducted on a pooled data set based on the average of the imputed values from the 4 assessments.

\section{Weight variability}

Weight variability was calculated with the use of the first 3 measured weights of the study at baseline, $6 \mathrm{wk}$, and $6 \mathrm{mo}$. Whereas previous studies of measured weight variability have used differing techniques to calculate weight variability $(21,22)$, the most common method has been to calculate the root mean square error of variation (RMSE) around the slope of weight change over time for each individual. The present study expanded on this idea by using growth curve analysis to calculate weight change trajectories over these 6 mo (23). This technique allows for linear regression curves to be modeled, taking into account both general trends across participants in weight over time and individual weight trends, rather than relying exclusively on the individual weight trajectory. The RMSE was then calculated around each participant's regression line. RMSE values ranged from 0.06 to $5.30(0.70 \pm 0.59)$. Weight variability as a predictor of future weight gain was assessed with the use of 2 linear regressions. The first examined weight variability as a predictor of weight change from month 6 to month 24. The second used a hierarchical linear model to assess contributions of potential covariates to the first linear model. Weight variability was calculated in $\mathrm{R}$ version 3.1.1 (Foundation for Statistical Computing). All other analyses were conducted with the use of SPSS (IBM Corporation) version 22.

\section{RESULTS}

To test whether weight variability over the first 6 mo predicted weight change over the subsequent $18 \mathrm{mo}$, a linear regression was conducted with weight change from month 6 to month 24 as the outcome variable and weight variability over the first 6 mo as the predictor variable. In this analysis, the assumption of homoscedasticity for weight variability was violated. The distribution of weight variability scores was not normal, but when an ln transformation of the variable was performed, it approached normality more closely and heteroscedasticity was no longer a major concern (skewness $\pm \mathrm{SE}:-0.44 \pm 0.19$; kurtosis $\pm \mathrm{SE}$ :
$0.79 \pm 0.37$ ). Therefore the $\ln$ transformation of weight variability was used in all regression analyses. Additionally, although several visual outliers appear in the scatterplot with the untransformed weight variability (Figure 1), they did not approach statistical significance as outliers per Cook's D (24) and were all deemed to be low in influence.

Weight variability significantly predicted weight change $(b \pm$ SE: $\left.1.47 \pm 0.43, P<0.01 ; R^{2}=0.06\right)$; the more participants' weight fluctuated, the more weight they gained later on (Figure 1). Weight variability and weight change during the initial 6-mo period were not associated, however $(b \pm \mathrm{SE}:-0.30 \pm 0.33$, $\left.P=0.37 ; R^{2}=0.01\right)$.

Next, hypothesized covariates were included in the model. These were the 3 baseline measures of eating behaviors (RRS, TEFQ-D, and DRES), baseline dieting status, baseline BMI, and 6-mo absolute weight change. RRS scores ranged from 2 to 28 (14.43 \pm 5.18$)$, TFEQ-D scores ranged from 1 to $13(6.78 \pm$ 3.12 ), and DRES scores ranged from 1.11 to 4.67 (2.77 \pm 0.73$)$. An independent samples $t$ test was used to compare weight variability between the intervention and control groups. Weight variability did not differ between groups $[t(169)=0.17, P=$ $0.86, d=0.03]$. Therefore, intervention condition was not included as a covariate in analyses. A hierarchical linear regression was conducted with all covariates included in block 1 and weight variability in block 2 to examine whether the addition of weight variability significantly improved the model. In block 1 , baseline BMI trended toward significantly predicting future weight change ( $b \pm \mathrm{SE}: 0.25 \pm 0.14, P=0.07)$. No other covariates were independently associated with future weight change. The inclusion of weight variability significantly improved the model $(P<0.01)$, increasing the $R^{2}$ value from 0.05 to 0.11 . In the full model, both baseline BMI and weight variability independently predicted weight gain (BMI still at a trend level) with control for dieting status, restrained eating, cognitive restraint, and disinhibition (Table 1). To control for the potential predictive value of changes in restraint or disinhibition over the initial 6 mo on future weight change, RMSE values around the regression lines of RRS, TFEQ-D, and DRES scores at baseline, $6 \mathrm{wk}$, and 6 mo were also calculated, tested as covariates in the relation between weight variability RMSE and future weight change. Variability in scores on the RRS, TFEQ-D, and DRES

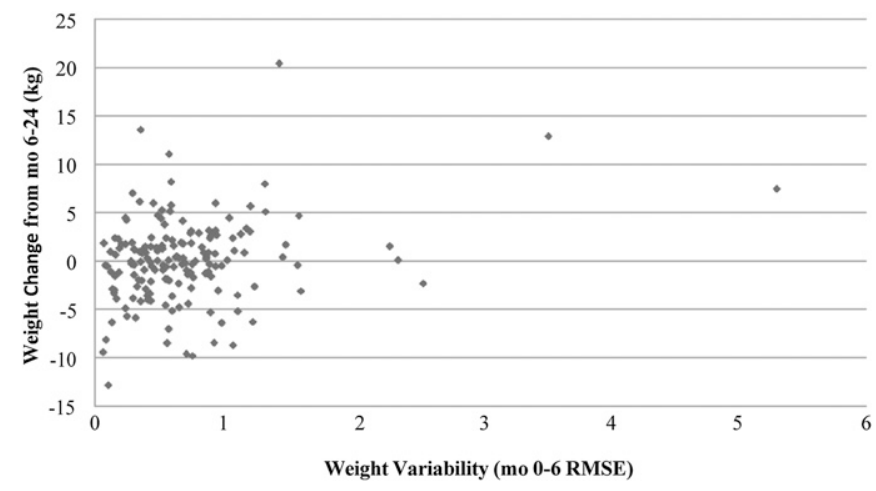

FIGURE 1 Association between 6-mo weight variability (RMSE) and subsequent 18-mo weight change. Linear regression revealed a significant positive relation between weight variability from baseline to 6-mo and subsequent 18 -mo weight change ( $b \pm \mathrm{SE}: 1.47 \pm 0.43, P<0.01 ; R^{2}=0.06$ ). The figure is a scatterplot illustrating this relation. RMSE, root mean square error of variation. 
TABLE 1

Linear regression model parameters as a predictor of 18-mo weight change $^{1}$

\begin{tabular}{|c|c|c|c|}
\hline Variable & $b$ & $\mathrm{SE}$ & $P$ \\
\hline RRS & -0.12 & 0.10 & 0.26 \\
\hline TFEQ-D & 0.03 & 0.13 & 0.85 \\
\hline DRES & -0.24 & 0.61 & 0.69 \\
\hline Dieting status $^{2}$ & -0.29 & 0.87 & 0.74 \\
\hline BMI & 0.25 & 0.13 & 0.06 \\
\hline 6-mo weight change & -0.12 & 0.11 & 0.26 \\
\hline Weight variability & 1.49 & 0.44 & $<0.01$ \\
\hline
\end{tabular}

${ }^{1} n=171$. DRES, Dutch Restrained Eating Scale; RRS, Revised Restraint Scale; TFEQ-D, Three-Factor Eating Questionnaire-Disinhibition subscale.

${ }^{2}$ Current diet to lose weight compared with no current diet or current diet to prevent weight gain.

were not significantly associated with subsequent 18-mo weight change, and the RMSE remained predictive when variability in these measures was controlled for.

Analysis of the imputed dataset replicated findings from the completer analyses; the RMSE continued to predict weight change when all covariates were controlled for $(b \pm \mathrm{SE}: 1.04 \pm 0.30$, $P<0.01)$. The only distinct finding in the imputed dataset was that 6-mo absolute weight change negatively predicted subsequent 18-mo weight change when RMSE and all other covariates were controlled for $(b \pm \mathrm{SE}:-0.20 \pm 0.06, P<0.01)$.

\section{DISCUSSION}

The results indicate that variation in weight over a 6-mo period predicted amount of weight gained over the subsequent 18 mo, independent of absolute weight change over the 6-mo period. It was reassuring that the same pattern of findings was obtained when all participants were included through the use of multiple imputation to account for missing follow-up weights. To our knowledge, this is the first demonstration that naturally occurring instability in humans' body weight over time predicts subsequent weight gain. Because the BMI of the sample studied was generally in the healthy weight range, the findings are relevant to the identification of individuals who are prone to weight gain but have not yet developed a substantial degree of overweight. The fact that the group studied was struggling with their weight was evident both in our participant selection criteria and in the fact that most participants gained or lost a substantial amount of weight over $2 \mathrm{y}$. Determining the degree to which this occurred because of the nature of the recruited sample (i.e., that they signed up for a weight gain prevention program) will require additional research.

There are 2 implications stemming from the selection criteria used in the present study. First, our results cannot necessarily be generalized to individuals who are less concerned about their weight or whose weight is more stable over time. Second, given that individuals who have weight concerns $(4,25)$ and frequent dieting attempts (3) are indeed liable to accelerated weight gain in the future, the current findings take on greater import because of the value of identifying early warning signs of future weight gain.

Because the prediction of weight gain by weight variability did not change when starting BMI or weight gain over the first 6 mo was controlled for, the present results do not appear to be a result of higher starting BMIs or higher weight gain in those showing the highest amount of weight variability over the first 6 mo. When measures of restrained eating, dieting, and disinhbition were included as covariates in the model, the predictive effect of weight variability did not change. None of these covariates themselves accounted for significant variance in long-term weight change other than baseline BMI. Furthermore, when variability in measures of disinhibition and restraint (assessed via RMSE) over the first 6 mo were used as covariates, the significant prediction of weight gain by weight variability was unchanged. Restrained eating/dieting, on the one hand, and disinhibition, on the other, could be viewed as capturing the tendency to restrict and lose control over eating, respectively, which in turn could produce increased weight variability. However, our results indicate that variation in body weight itself had superior predictive ability relative to specific eating patterns that could contribute to weight fluctuations. One explanation for these findings is that self-report measures of food intake often are insufficiently valid as measures of naturalistic energy intake $(26,27)$.

Our measurement of weight at 3 time points over 6 mo was dictated by the design of the original intervention, not by any theoretical consideration. Future research will be needed, not only to examine the replicability of these results but also to test different lengths of time during which weights are assessed and different numbers of measured weights to assess variability. It is likely that the period during which weight is measured would need to be sufficiently long to permit meaningful variations in body weight to manifest themselves.

It is important to distinguish between the weight variability studied here from the topic of weight cycling or yo-yo dieting studied in the past. The past focus on weight cycling has been based on the idea that volitional weight loss is often followed by weight regain and, over time, a history of repeated weight fluctuations (28). There is some evidence that weight cycling predicts future weight gain (29) and cardiometabolic disease (30). However, the current study focused on naturally occurring weight variability in young women mostly in the healthy weight range rather than the consequences of repeated intentional weight losses. Future research should examine whether greater weight fluctuation in individuals in the healthy weight range represents a health risk independently of body mass or change in body mass (30).

The current results have both practical and conceptual implications. If the results can be replicated, then variability in body weight could be used as a potential early warning of future weight gain while there is time to intervene to prevent it. Many people weigh themselves infrequently and therefore have little awareness of small changes in their weight. Furthermore, a person who was experiencing greater weight gains and losses might not realize that these bidirectional changes may mean that his or her body's ability to automatically maintain a steady state is deteriorating. If a simple system were available to periodically inform people of their degree of weight variability, it might serve as a useful cue to examine how changes in their food intake or physical activity might be contributing to these changes.

Conceptually, set-point theory has long noted that the body mass of individuals shows remarkable stability (indicating that short-term energy surfeits and deficits are reliably and accurately compensated for) over long periods despite an energy flux of millions of calories (31). Perhaps an early sign of the degradation of this highly calibrated system of weight control is an increase in 
body weight variability. Three potentially interrelated explanations for the prediction of long-term weight gain by weight variability are the following: 1) the obesogenic environment continuously drives energy balance in a positive direction; 2) freshmen predisposed (by genetic or other factors) toward weight gain begin to manifest their vulnerability in the novel college environment; and 3) individuals most prone to sustained positive energy balance counteract weight gains by following weight loss diets but ultimately fail to prevent their underlying predisposition toward weight gain from manifesting itself. However, given that controlling for restrained eating and disinhibition-measured either statically at baseline or dynamically over 6 mo-did not change the prediction of weight gain by weight variability, the sources of individual differences in variability in body weight over time remain unclear.

Strengths of this study include its relatively large sample size of weight-concerned women and the fact that objectively measured weights were obtained over a 2-y span. We were also fortunate that our sample exhibited high degrees of weight variability over time, because the ability to identify predictors of weight change depends in part on sufficient variability in the predictor of interest. Weaknesses of the study included the inclusion of women only and the fact that they were observed at a unique time of their lives (i.e., the beginning of college). The fact that a substantial proportion of those who initially joined the study did not contribute body weights at all assessment periods was also a weakness.

In sum, to our knowledge, the current study was the first to find that variability of body weight over an initial time period predicted weight gain over a longer time span. This significant prediction was maintained when beginning body mass, change in body mass over the period during which weight variability was assessed, disinhibition, restraint, and dieting were controlled. The present results are open to differing interpretations, but one intriguing possibility is that increased variation in body weight comprises a weakening of the typically finely tuned ability of humans to automatically and accurately maintain energy balance and body weight over long periods of time.

The authors' contributions were as follows-MRL and EHF: designed the weight variability research study, conducted the research, and had primary responsibility for the final content of the manuscript; MRL and ES: provided the database; MRL, EHF, and ES: analyzed the data or performed the statistical analysis; and MRL, EHF, SRW, and ES: wrote the manuscript. None of the authors reported a conflict of interest related to the study.

\section{REFERENCES}

1. Keesey RE, Hirvonen MD. Body weight set-points: Determination and adjustment. J Nutr 1997;127:1875S-83S.

2. Chernyak Y, Lowe MR. Motivations for dieting: Drive for thinness is different from drive for objective thinness. J Abnorm Psychol 2010; 119:276-81.

3. Lowe MR, Doshi SD, Katterman SN, Feig EH. Dieting and restrained eating as prospective predictors of weight gain. Front Psychol 2013;4:577.

4. van den Berg P, Neumark-Sztainer D. Fat'n happy 5 years later: Is it bad for overweight girls to like their bodies? J Adolesc Health 2007;41:415-7.

5. Lowe MR, Annunziato RA, Markowitz JT, Didie E, Bellace DL, Riddell L, Maille C, McKinney S, Stice E. Multiple types of dieting prospectively predict weight gain during the freshman year of college. Appetite 2006;47:83-90.

6. Stice E, Marti CN, Spoor S, Presnell K, Shaw H. Dissonance and healthy weight eating disorder prevention programs: long-term effects from a randomized efficacy trial. J Consult Clin Psychol 2008;76:329.

7. Lowe MR, Tappe KA, Annunziato RA, Riddell LJ, Coletta MC, Crerand CE, Didie ER, Ochner CN, McKinney S. The effect of training in reduced energy density eating and food self-monitoring accuracy on weight loss maintenance. Obesity (Silver Spring) 2008;16:2016-23.

8. Lowe MR. Self-regulation of energy intake in the prevention and treatment of obesity: Is it feasible? Obes Res 2003;11 Suppl:44-59.

9. Pietrobelli A, Faith MS, Allison DB, Gallagher D, Chiumello G, Heymsfield SB. Body mass index as a measure of adiposity among children and adolescents: a validation study. J Pediatr 1998;132: 204-10.

10. Stunkard AJ, Messick S. The three-factor eating questionnaire to measure dietary restraint, disinhibition and hunger. J Psychosom Res 1985;29:71-83.

11. Laessle RG, Tuschl RJ, Kotthaus BC, Prike KM. A comparison of the validity of three scales for the assessment of dietary restraint. J Abnorm Psychol 1989;98:504-7.

12. Weinstein SE, Shide DJ, Rolls BJ. Changes in food intake in response to stress in men and women: Psychological factors. Appetite 1997;28:7-18.

13. Hays NP, Roberts SB. Aspects of eating behaviors "disinhibition" and "restraint" are related to weight gain and bmi in women. Obesity (Silver Spring) 2008;16:52-8.

14. van Strien T, Frijters JER, van Staveren WA, Defares PB, Deurenberg P. The predictive validity of the Dutch Restrained Eating Scale. Int J Eat Disord 1986;5:747-55.

15. Lowe MR, Thomas JG. Measures of restrained eating: conceptual evaluation and psychometric update. In: In Allison D, Baskin ML, editors. Handbook of assessment methods for obesity and eating behaviors. New York: Sage; 2009. p. 137-85.

16. Stice E, Sysko R, Roberto CA, Allison S. Are dietary restraint scales valid measures of dietary restriction? Additional objective behavioral and biological data suggest not. Appetite 2010;54:331-9.

17. Heatherton TF, Polivy J, Herman CP. Restraint, weight loss, and variability of body weight. J Abnorm Psychol 1991;100:78.

18. Polivy J, Herman CP, Howard KI. The Restraint Scale: assessment of dieting. In: Hersen M, Bellack A, editors. Encyclopedia of assessment devices. New York: Pergamon Press; 1988. p. 377-80.

19. Allison DB, Kalinsky LB, Gorman BS. A comparison of the psychometric properties of three measures of dietary restraint. Psychol Assess 1992;4:391-8.

20. Ruderman AJ. Dysphoric mood and overeating: A test of restraint theory's disinhibition hypothesis. J Abnorm Psychol 1985;94:78-85.

21. French S, Jeffery R, Folsom A, Williamson D, Byers T. Weight variability in a population-based sample of older women: reliability and intercorrelation of measures. Int J Obes Relat Metab Disord 1995;19(1):22-9.

22. Vergnaud A-C, Bertrais S, Oppert J-M, Maillard-Teyssier L, Galan P, Hercberg S, Czernichow S. Weight fluctuations and risk for metabolic syndrome in an adult cohort. Int $\mathrm{J}$ Obes (Lond) 2008;32:315-21.

23. Mirman D. Growth curve analysis and visualization using R. Boca Raton (FL): CRC Press; 2014.

24. Cook RD, Weisberg S. Residuals and influence in regression. New York: Biometrical Journal; 1982.

25. Haines J, Neumark-Sztainer D, Wall M, Story M. Personal, behavioral, and environmental risk and protective factors for adolescent overweight. Obesity (Silver Spring) 2007;15:2748-60.

26. Lichtman SW, Pisarska K, Berman ER, Pestone M, Dowling H, Offenbacher E, Weisel H, Heshka S, Matthews DE, Heymsfield SB. Discrepancy between self-reported and actual caloric intake and exercise in obese subjects. N Engl J Med 1992;327:1893-8.

27. Mertz W, Tsui JC, Judd JT, Reiser S, Hallfrisch J, Morris ER, Steele $\mathrm{PD}$, Lashley E. What are people really eating? The relation between energy intake derived from estimated diet records and intake determined to maintain body weight. Am J Clin Nutr 1991;54:291-5.

28. Brownell KD, Steen SN, Wilmore JH. Weight regulation practices in athletes: analysis of metabolic and health effects. Med Sci Sports Exerc 1987;19:546-56.

29. Kroke A, Liese A, Schulz M, Bergmann M, Klipstein-Grobusch K, Hoffmann K, Boeing H. Recent weight changes and weight cycling as predictors of subsequent two year weight change in a middle-aged cohort. Int J Obes Relat Metab Disord 2002;26(3):403-9.

30. Montani JP, Schutz Y, Dulloo AG. Dieting and weight cycling as risk factors for cardiometabolic diseases: who is really at risk? Obes Rev 2015;16(Suppl 1):7-18.

31. Speakman JR, Levitsky DA, Allison DB, Bray MS, de Castro JM, Clegg DJ, Clapham JC, Dulloo AG, Gruer L, Haw S, et al. Set points, settling points and some alternative models: theoretical options to understand how genes and environments combine to regulate body adiposity. Dis Model Mech 2011;4:733-45. 